\title{
FIXED POINTS FOR CONFLUENT MAPS ONTO DISKS
}

\author{
SAM B. NADLER, JR.
}

\begin{abstract}
Let $M$ be a compact subset of a disk $D$ such that $H^{1}(M) \approx 0$. It is shown that if $f$ is a confluent mapping from $M$ onto $D$ and if $g$ is any mapping from $M$ into $D$, then $f(p)=g(p)$ for some $p \in M$.
\end{abstract}

Let $R^{2}$ denote the Euclidean plane and let $B^{2}=\left\{\left(x_{1}, x_{2}\right) \in R^{2}: x_{1}^{2}+x_{2}^{2}\right.$ $<1$ \}. In [6] Hamilton proved the following theorem: If $f$ is an open mapping from a locally connected unicoherent continuum $M \subset B^{2}$ onto $B^{2}$, then $f$ has a fixed point. The following theorem improves Hamilton's theorem by allowing more general mappings $f$, by not requiring that $M$ be locally connected, and by obtaining a much stronger conclusion. Recall that a mapping (= continuous function) $f$ from a space $Y$ onto a space $Z$ is confluent $[1$, p. 213] provided that for each compact connected subset $K$ of $Z$ and each component $A$ of $f^{-1}(K)$, we have $f[A]=K$. A mapping $f: Y \rightarrow Z$ is universal [7, p. 433] provided that if $g$ is any mapping from $Y$ into $Z$, then there exists $p \in Y$ such that $f(p)=g(p)$.

THEOREM. Let $M$ be a compact subset of $B^{2}$ such that $M$ does not separate $R^{2}$. If $f: M \stackrel{\text { onto }}{\rightarrow} B^{2}$ is confluent, then $f$ is universal (and, thus, $f$ has a fixed point).

Proof. Let $S^{1}=\left\{\left(x_{1}, x_{2}\right) \in R^{2}: x_{1}^{2}+x_{2}^{2}=1\right\}$. Let $M_{1}=f^{-1}\left(S^{1}\right)$ and let $f_{1}=f \mid M_{1}$ (the restriction of $f$ to $M_{1}$ ). Note that, since $f$ is confluent, $f_{1}$ : $M_{1} \stackrel{\text { ont }}{\rightarrow} S^{1}$ is confluent. If follows from a theorem in [9, p. 229] that any confluent mapping from a compact Hausdorff space onto $S^{1}$ is essential (= not nullhomotopic)-see Remark 2 here. Hence, $f_{1}$ is essential. Thus, since every mapping from $M$ into $S^{1}$ is nullhomotopic (see $[3, \cdot 2.1$, p. 357]), we see that $f_{1}$ cannot be extended to a mapping of $M$ into $S^{1}$. Therefore, by the lemma in [11], $f$ is universal (and hence, by using the inclusion map $g$ : $M \rightarrow B^{2}$, we see that $f$ has a fixed point since $f(p)=g(p)$ for some $\left.p \in M\right)$.

Of special interest in the following corollary is the fact that Hamilton's theorem remains valid for monotone mappings.

Received by the editors November 7, 1978 and, in revised form, February 8, 1979.

AMS (MOS) subject classifications (1970). Primary 54H25, 54F20; Secondary 54F60.

Key words and phrases. Confluent map, essential map, fixed point, monotone map, open map, quasi-interior map, universal map. 
Corollary. Let $M$ be a compact subset of $B^{2}$ such that $M$ does not separate $R^{2}$. If $f: M \stackrel{\text { onto }}{\rightarrow} B^{2}$ is a monotone, open, or quasi-interior mapping, then $f$ is universal (and, thus, $f$ has a fixed point).

Proof. Since these types of mappings are confluent (see [1, p. 214] and [10, 2.7]), the corollary follows from the theorem.

REMARK 1. Let us note that the proof of our theorem yields the following more general fact: Let $Y$ be a compact Hausdorff space such that $Y$ is $\operatorname{cr}\left(S^{1}\right)$ $[8, p .434]$ or, equivalently, such that $H^{1}(Y) \approx 0$. If $f: Y \stackrel{\text { onto }}{\rightarrow} B^{2}$ is confluent, then $f$ is universal.

REMARK 2. In the proof of our theorem, we used a special case of a theorem in [9, p. 229]. This special case has the following easy proof. Let $k$ be a confluent mapping from a compact Hausdorff space $X$ onto $S^{1}$. Suppose that $k$ is nullhomotopic. Then [4, 5.3, p. 18], $k=e^{i \psi}$ for some mapping $\psi$ : $X \rightarrow R^{1}$ (the reals). Let $g=e^{i} \mid \psi[X]$. Since $k$ is confluent and $k=g \circ \psi, g$ is confluent. Let $A$ be a component of $g^{-1}\left(S^{1}\right)$ and let $g^{\prime}=g \mid A$. Since $g$ is confluent, $g^{\prime}: A \stackrel{\text { onto }}{\rightarrow} S^{1}$ is confluent and, since $X$ is compact, $A$ is a bounded closed interval. Therefore, we have a contradiction to [2, Corollary 20, p. 32] which says that a confluent image of an arc is an arc (or a point). The reader may wish to see [5] for generalizations of results in [9] and an affirmative answer to Problem 558 in [9, p. 233].

REMARK 3. It is clear that every mapping from an arc onto a larger arc has a fixed point (this is also true for mappings from any chainable continuum onto a larger chainable continuum). However, for $n>2$, there are fixedpoint-free mappings from $n$-cells onto larger $n$-cells [12].

The question of whether Hamilton's theorem could be generalized to confluent mappings was raised by Carl Eberhart in a conversation with the author.

ADDED IN PROOF. Recently the author has generalized the results in this paper to weakly confluent mappings. The manuscript, entitled Universal mappings and weakly confluent mappings, has been submitted for publication; an abstract of some of the results is in the Notices Amer. Math. Soc. 26 (1979), 79T-G80, p. A-445.

\section{BIBLIOGRAPHY}

1. J. J. Charatonik, Confluent mappings and unicoherence of continua, Fund. Math. 56 (1964), 213-220.

2. __ On fans, Dissertationes Math. 54 (1967).

3. James Dugundji, Topology, Allyn and Bacon, Boston, Mass., 1966.

4. Marvin J. Greenberg, Lectures on algebraic topology, Benjamin, New York, 1967.

5. J. Grispolakis and E. D. Tymchatyn, Semi-confluent mappings and acyclicity, Houston J. Math. 4 (1978), 343-357.

6. O. H. Hamilton, Fixed point theorems for interior transformations, Bull. Amer. Math. Soc. 54 (1948), 383-385.

7. W. Holsztyński, Universal mappings and fixed point theorems, Bull. Acad. Polon. Sci. Sér. Sci. Math. Astronom. Phys. 15 (1967), 433-438.

8. K. Kuratowski, Topology, vol. II, Academic Press, New York, 1968. 
9. A. Lelek, On confluent mappings, Colloq. Math. 15 (1966), 223-233.

10. A. Lelek and David R. Read, Compositions of confluent mappings and some other classes of functions, Colloq. Math. 29 (1974), 101-112.

11. O. W. Lokuciewski, On a theorem on fixed points, Uspehi Mat. Nauk (N. S.) 12 (1957), no. 3 (75), 171-172. (Russian)

12. Sam B. Nadler, Jr., Examples of fixed point free maps from cells onto larger cells and spheres (submitted).

Department of Mathiematics, Universtty of Kentucky, LeXington, Kentucky 40506

Current address: Department of Mathematics, West Virginia University, Morgantown, West Virginia 26506 